\title{
UWB Reactive Near Field Detection for Smart Packages: A Comprehensive Study using eggs
}

\author{
Heng Ma, Tim W. C. Brown, Member, IEEE, and Anthony T. S. Ho, Senior Member, IEEE
}

\begin{abstract}
Detection of packages or storage containers relies heavily on the use of radio frequency identification (RFID) tagging, though such technology provides no means to determine the quantity of items within them. This paper presents a comprehensive study of ultra wideband (UWB) detection in the reactive near field as a low cost, low power way of detecting solid items within a package to complement RFID. For proof of concept, egg boxes within a smart fridge are used as a chosen test case. Simulations and measurements are carried out to evaluate the filtering of the UWB impulse response from which it can resolve the quantity of eggs in a box, using an array of sensors either attached to the package or placed underneath. Correlation coefficients are derived as a metric of this filtering in a reactive near field detection scenario. The robustness of the approach is further evaluated by considering other food cluttered around and above the egg box. The results show smart packages which detect an item directly above the sensor are not affected by surrounding clutter.
\end{abstract}

Index Terms - Reactive near field, ultra wideband (UWB), smart fridge, Internet of things (IoT), pulse filtering

\section{INTRODUCTION}

S MART packages can be considered as passive 'things', which can be connected to the Internet of Things (IoT), with significant applications including supply chain management, logistics and health/pharmaceutical supplies. Connection to the package is realized by a radio frequency identification (RFID) system where a package can have an RFID tag attached to it containing product information [1]. However, an RFID tag is unable to determine the number of items in a package, which requires a new enabling technology. Reactive Near Field ultra wideband (UWB) detection is investigated in this paper as a candidate technology, which could be adapted to RFID technology, either as an extra radio to be powered passively like an RFID tag or to act as a smart shelf to assist RFID tagged packages.

In order to study the reliability of the concept, it was necessary to choose a suitable example application in this paper, where a smart egg box for use in an intelligent fridge (iFridge) was selected. The iFridge as an IoT solution could have significant benefits in many use cases including domestic [2]-[3], healthcare [4]-[5] and logistics [6]. A smart package with the ability to determine the quantity of items contained within it would be beneficial to content

H. Ma and T. W. C. Brown are with the Institute for Communication Systems, University of Surrey, Guildford, GU2 7XH, UK (email:tim@brown76.name).

A. T. S. Ho is with the Department of Computer Science, University of Surrey, Guildford, GU2 7XH, UK and visiting professor at Wuhan University of Technology and Tianjin University of Science and Technology, China (Email: a.ho@surrey.ac.uk). management and intelligent systems. An iFridge containing items such as eggs may use an "egg minder" for content awareness by placing the eggs in a dedicated tray with switch detectors. However, this comes with limitations; most notably the user must remove the eggs from the box, possibly losing important information such as the expiration date. Smart packaging technology would therefore be a significant advance.

Appreciable research efforts have been invested into the detection of targets for UWB medical imaging including the localization of malignant breast tissue [7], through wall [8] and ground penetrating radar [9]. The main advantage of UWB imaging based detection is the high resolution due to the large bandwidth. Non-imaging UWB target detections have been conducted where promising techniques fall into two categories: resonant [10] and polarimetric [11] based approaches. The natural resonant frequency from late time response caused by the creeping wave was evaluated for detection in previous research such as military aircraft models [12] and shapes [13]. Owing to the overlap of resonant frequencies, the resonant approach lacks the ability to know the quantity of eggs [14]. The polarimetric based approach uses the complex residues of the polarization independent natural poles [15]-[17], in which characteristic polarization states (CPSs) including the tilt angle, characteristic angle and ellipticity are evaluated under the illumination of different polarization states [15]. The number of eggs were found to be retrieved in terms of CPSs reported in [14], revealing that the quantity is proportional to the characteristic angle. However, three limitations arose. Firstly, in order to sense the eggs using the polarimetric approach, the eggs are required to be illuminated using plane wave, thus far field criteria is necessary. Secondly, the distance between sensors and the eggs are critical in order to avoid the collision of the transmitted waves and scattered waves thus enabling a late time response. Finally, in a conventional UWB sensor covering 3 to $10 \mathrm{GHz}$, the illuminated pulse width is $0.286 \mathrm{~ns}$ requiring a minimum distance of $8.5 \mathrm{~cm}$ to allow sufficient propagation time.

Content awareness technologies for detecting eggs in an iFridge require the ability to detect close to the eggs at which point the items concerned are placed in the reactive near field because the sensors are either attached to the egg box or it is brought into contact with the sensors on the shelf. Information of the detected quantity would be relayed using a sensor node, which can be developed using an active or even passive RFID chip whereby the low power UWB radio could be excited in such a way. Therefore the novelty of the work presented in this paper builds on results reported in [18] to demonstrate the ability of UWB in the reactive near field to 
detect objects, which will filter the impulse response when close to the sensor and cannot be considered as radar. For eggs in an iFridge, their presence and position in a box placed on top of sensors are determined using a derived correlation coefficient for purposes of analyzing the full effect of filtering, since only partial information is obtained from the conventional group delay. A UWB antenna array covering the band of $3-10 \mathrm{GHz}$ was fabricated to conduct exhaustive measurements, within which the $3-5 \mathrm{GHz}$ sub band is found to be of interest, which requires a lower sampling rate at the receiver thus reducing system complexity and cost.

The paper is organized as follows. Section II introduces the simulation and measurement setup involving egg detection as well as the derived correlation coefficient metric used. Both simulations and measurements of egg quantity detection in a clutter free environment are presented in Section III. Followed by this, scenarios with clutter are taken into account in Section IV. Conclusions are reached in the final section, where consideration is given to items in a package other than eggs followed by an appendix.

\section{SYSTEM CONCEPT AND MEASUREMENT SETUP}

The system concept used in this paper as well as the simulation and measurements carried out to validate the concept are described in this section. First the system architecture is shown, which is subsequently followed by details of the measurement setup, simulation parameters and finally the metrics derived for analysis.

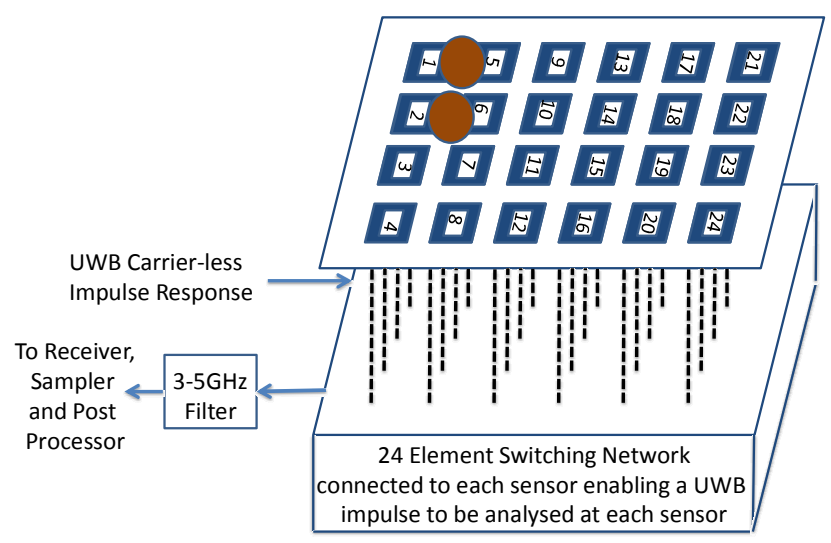

Fig.1 Illustration of the system architecture for a shelf in a smart fridge. Each sensor is numbered 1 to 24

\section{A. System Concept for egg detection}

A schematic diagram of the system concept for a smart fridge scenario is drawn in Fig. 1. An array of 24 sensors in this case (though there could be more or less of these depending on the size of a shelf in a smart fridge) is used and connected to a switching network, which would allow a UWB impulse to be transmitted from any one sensor and then received by that same sensor (for monostatic detection) or any other sensor (for bistatic detection). The filter is subsequently used to narrow the bandwidth of the signal to that of the low complexity receiver and sampler from 3-5 GHz, which enables post processing. The post processing will include a Fast Fourier Transform to enable the data to be analyzed in the frequency domain. In Fig. 1, two eggs (which could also be contained in an egg box and positioned next to each other) are shown as an example placed in the vicinity of sensors 1,2, 5 and 6 . The analysis in this paper shows how the egg quantity can be detected regardless of their position relative to the sensors in their vicinity. Up to four eggs will be considered, which could extend to more eggs using the same concept.

\section{B. UWB sensors used for simulation and measurement}

A total number of 24 sensors in a 4 × 6 planar array were fabricated as shown in Fig. 2. The total array dimension is 210 $\mathrm{mm} \times 297 \mathrm{~mm}$. The center to center distances between adjacent elements in the $x$ and $y$ directions are $50 \mathrm{~mm}(0.5 \lambda$ at $3 \mathrm{GHz})$ and $45 \mathrm{~mm}(0.4 \lambda$ at $3 \mathrm{GHz})$ respectively.
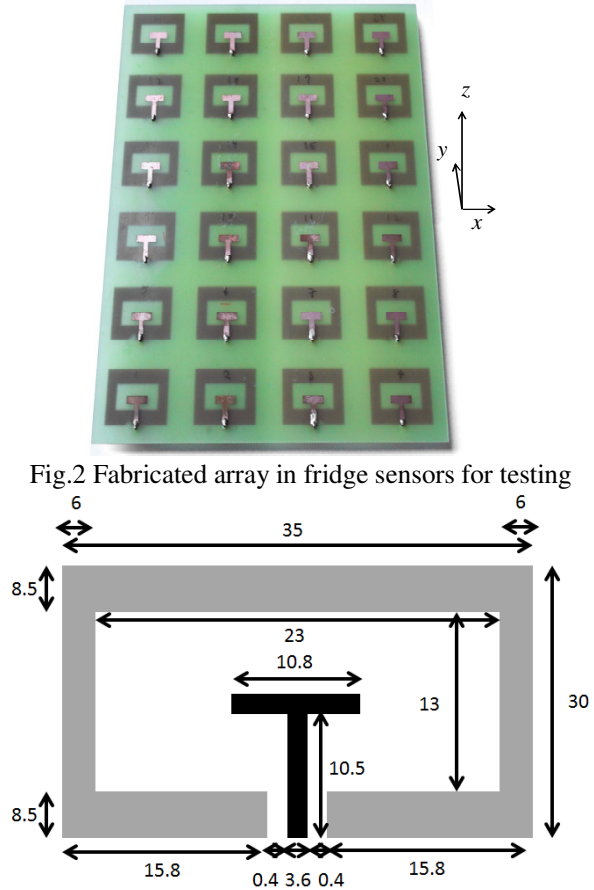

Fig. 3 Geometry of antenna element with grey conductor printed on the bottom side of the substrate, black on the front side (not to scale for clarity)

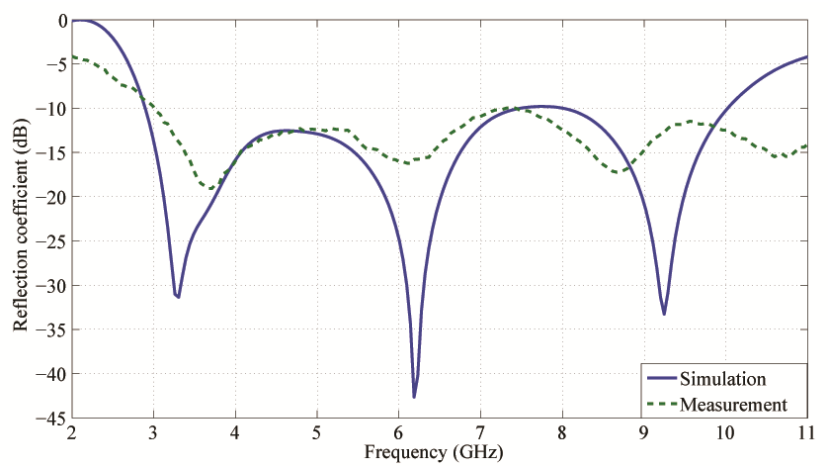

Fig.4 Simulated and measured reflection coefficient of in fridge sensor

For each sensor, a conventional coplanar waveguide fed UWB planar monopole antenna [19] (Fig. 3 (a)) is chosen for this study with dimensions $30 \times 35 \times 1.6 \mathrm{~mm}$. While the antenna design would allow light to pass through to help illuminate a fridge, such antennas in future systems, along with the system architecture, could be implemented with transparent conducting materials such as graphene or carbon 
nanotubes [20], thus being invisible and implementable on any item employing content-awareness. For measurement with a vector network analyzer, FR4 is used as a substrate for the proposed antenna with a dielectric constant of 4.55 , while dimensions shown in Fig. 3 are in $\mathrm{mm}$. The fabricated design used in this instance is modified to have a grounded waveguide feed so that the ground element and coaxial connector is on the bottom side of the substrate as indicated in grey in Fig. 3 thus allowing coaxial connectors to be attached easily to the bottom side. Good agreement between the simulated and measured reflection coefficient is shown from 3 to $10 \mathrm{GHz}$ in Fig. 4 where the return loss exceeds $10 \mathrm{~dB}$.

\section{Egg Model for Simulation}

The egg used in the simulation can be considered as an ellipsoid having a maximum radius of $2 \mathrm{~cm}$ and height of 5 $\mathrm{cm}$. Besides its physical geometry, the dielectric constant, $\varepsilon_{r}$ is required. Extensive studies of the dielectric constant of eggs have been conducted [21]-[23], however the frequencies of interest were in the range from 10 to $1800 \mathrm{MHz}$. The dielectric constant of eggs in UWB can be estimated in terms of curve fitting with the relevant studies shown in [18].

\section{Correlation coefficient metric}

The presence of eggs above a sensor in the reactive near field causes a filtering of the UWB impulse, while also the reflection coefficient response of the sensors is changed by the eggs, thus changing the magnitude of the S-parameters. In addition, the filtering effect will lead to a change in group delay, which is defined as follows [25]:

$$
\tau_{g}=-\frac{\partial}{\partial \omega} \angle H(\mathrm{j} \omega)
$$

where $\angle H(\mathrm{j} \omega)$ is the phase response of a system derived from the $\mathrm{S}$-parameters dependent on frequency $\omega$.

A measurement example of the reflection coefficient, $s_{11}$, when the sensor is covered and uncovered (free space) by the egg is plotted in Fig. 5. In comparison to free space, the most resonant frequency changes from $3.8 \mathrm{GHz}$ to $4 \mathrm{GHz}$ as the egg is placed above the sensor due to the EM coupling and detuning. The corresponding group delay is evaluated in Fig. 6 , where a low group delay always below $1.2 \mathrm{~ns}$ is observed in the free space case. However, a significant change in the maximum group delay due to the presence of the egg can be observed as a spike at $4 \mathrm{GHz}$, where the reflection coefficient resonates. This is due to a rapid phase change as the reflection coefficient reduces towards a null point at this frequency and the signal is largely absorbed by the egg. Group delay evaluates only one aspect of filtering of the impulse response and as such it does not change dramatically over the whole band except at the resonant frequency. Therefore, to account for the change in magnitude over the whole band (which is more significant) as well as phase variation, a complex correlation coefficient metric is derived in this paper, which can also be applied as a quantitative analysis on how the eggs filter the UWB impulse response. The reflection coefficient correlation (RCC), which is a measure of the effect of the presence of an egg in the monostatic case can be expressed as: $\rho_{\text {RxTxi }}=\frac{E\left[S_{\text {RxTxi_egg }} S_{\text {RxTxi_free }}^{*}-E\left[S_{\text {RxTxi_egg }}\right] E\left[S_{\text {RxTxi_free }}^{*}\right]\right.}{\sqrt{\sigma^{2}\left|S_{\text {RxTix_egg }}\right| \sigma^{2} \mid S_{\text {RxTix_free }}}}$

where $\mathrm{S}_{\mathrm{Rx} i \mathrm{Tx} i \text { egg }}$ denotes the $\mathrm{S}$-parameter for sensor $i$ (i.e. $s_{i i}$ ) when the eggs are present, while $S_{\text {RxiTxi free }}$ denotes parameter $s_{i i}$ in the free space case when eggs are not present. The superscript symbol ${ }^{*}$ is the complex conjugate. The variance of $S_{\mathrm{Rx} i \mathrm{~T} x i_{-} \text {free }}$ and $\mathrm{S}_{\mathrm{Rx} i \mathrm{Tx} i_{-} \text {egg }}$ is defined by:

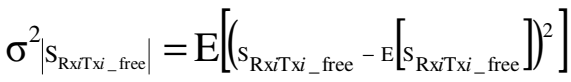

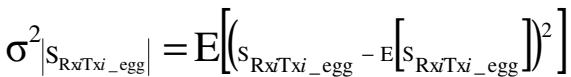

where the averaging using the expected value function $\mathrm{E}[]$, is calculated by taking all of the frequency bins. S-parameter data is measured over the frequency range of $2 \mathrm{GHz}$ to 11 $\mathrm{GHz}$ using 201 frequency bins, within which the data of 45 frequency bins from $3 \mathrm{GHz}$ to $5 \mathrm{GHz}$ is extracted for evaluation. The simulated results also use the same frequency bin size.

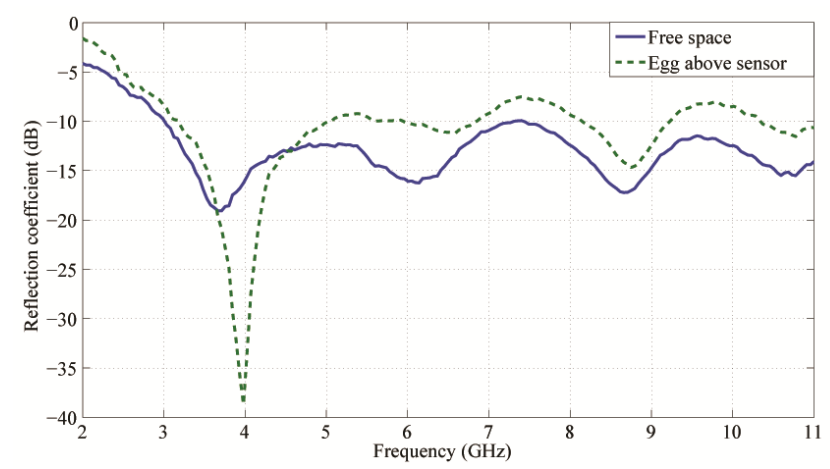

Fig.5 Measured reflection coefficient in the case of free space and an egg above sensor

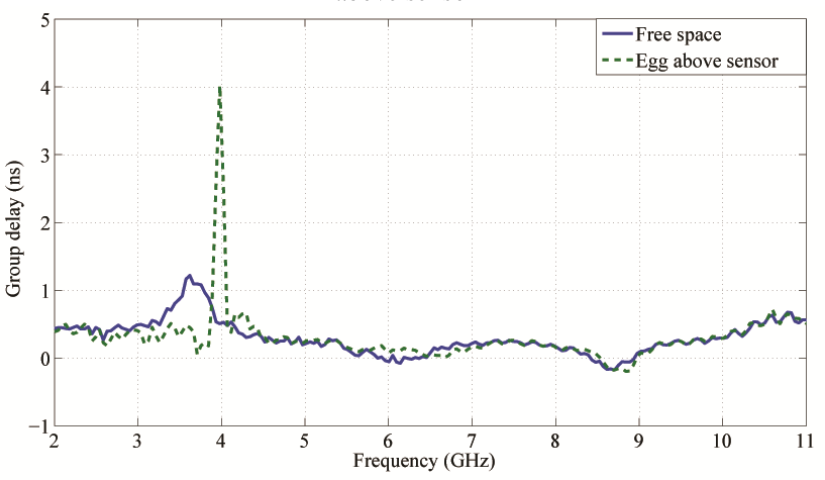

Fig.6 Measured group delay in the case of free space and an egg above sensor

The coupling coefficient correlation (CCC) is also evaluated as a measure of the effect of the presence of the eggs in the bistatic case, which can be expressed as:

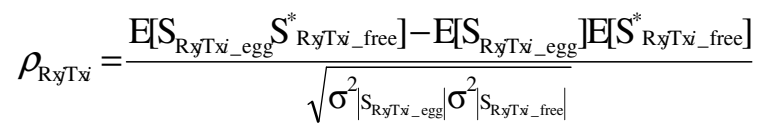

where $\mathrm{S}_{\mathrm{Rx} j \mathrm{~T} x i_{\text {egg }}}$ denotes the S-parameter between transmit sensor $i$ and receive sensor $j$ (or $s_{j i}$ ) when the eggs are present, 
while $\mathrm{S}_{\mathrm{Rx} j \mathrm{~T} i{ }_{-} \text {free }}$ denotes parameter $s_{j i}$ in the free space case when eggs are not present. The variance of $\mathrm{S}_{\mathrm{Rx} j \mathrm{Tx} i \text { egg }}$ and $\mathrm{S}_{\mathrm{Rxj} \text { Txi_free }}$ are defined in the same manner as equations (3) and (4).

\section{EGG DETECTION IN A RANDOM POSITION}

Simulations carried out in [18] determined the detectable region of the sensors in the reactive near field. The results found that the maximum distance from the centre of the sensor was $25 \mathrm{~mm}$ in the $x$ and $y$ directions at which detection could be achieved due to a change in RCC. Also as a preliminary study, the eggs were placed directly above the corresponding sensors, where it was found the real part of the RCC dropped to a value as low as -0.4 due to the presence of eggs, while the imaginary part was always less than 0.25 and considered negligible compared to the change in the real part. The CCC for the case where only one egg is directly placed above six different sensors was also studied, where it was found that only sensors adjacent to the egg had any significant information. This paper extends the study further by taking an empirical set of measurements to evaluate the robustness of the reactive near field detection method, regardless of the position of the eggs relative to the sensors.

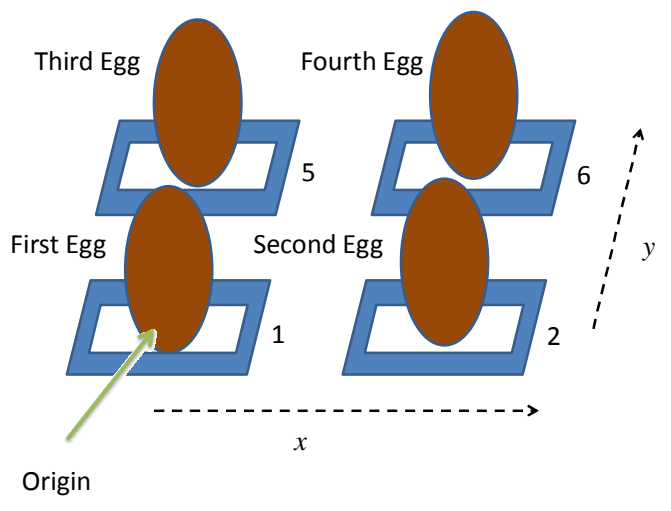

Fig.7 Coordinates for study of eggs in random positions

In this study, a set of four sensors: 1, 2, 5 and 6 of the array in Fig. 1, illustrated in Fig.7, are used to detect the presence of up to four eggs, which at first are placed directly over their respective sensor. Taking the origin $(0,0)$ as directly above sensor 1 , the eggs are translated in steps of $5 \mathrm{~mm}$ denoting the horizontal $x$ direction and vertical $y$ direction to be relative to this origin as illustrated in Fig. 7. Also labelled in Fig. 7 are the first, second, third and fourth eggs, which indicate the starting positions when 1 to 4 eggs are tested in this study. The total distance each egg moves either in the $x$ or $y$ direction is $50 \mathrm{~mm}$ since at this point an egg will cover the next sensor above or adjacent to its original one.

Fig. 8 illustrates in the blue and green curves, the measured RCC of sensor 1 both when the egg moves in the $x$ direction at a step size of $5 \mathrm{~mm}$ while fixing the $y$ direction at $0 \mathrm{~mm}$ and when the egg moves in the $y$ direction at a step size of $5 \mathrm{~mm}$ while fixing the $x$ direction at $0 \mathrm{~mm}$. Under the same test conditions, the CCC is plotted in the red and turquoise curves between sensors 1 and 2 when $y$ is fixed and sensors 1 and 5 when $x$ is fixed. As shown, the RCC rises above 0.7 after moving $21 \mathrm{~mm}$ in the $x$ direction. The CCC of sensor 1 and 2 is as high as 0.89 at the start, while reaching a minimum of 0.7 when the egg moves away $20 \mathrm{~mm}$. The CCC of sensor 1 and 5 has a similar trend of that for sensor 1 and 2 though it falls below 0.6 when moved $20 \mathrm{~mm}$. A coherence distance, defined as a measure of the distance at which the correlation falls from the maximum starting value to a threshold of 0.7 , is found from Fig. 8 to be $30 \mathrm{~mm}$ for the RCC regardless of either varying $x$ or $y$ directions, while for the CCC it is $20 \mathrm{~mm}$ in the $x$ direction and $10 \mathrm{~mm}$ in the $y$ direction. The step size the eggs are moved by is $5 \mathrm{~mm}$, which is $50 \%$ or less compared to the coherence distance, thus providing sufficient resolution for analysis in this paper. The imaginary part of the correlation is ignored due to negligible values.

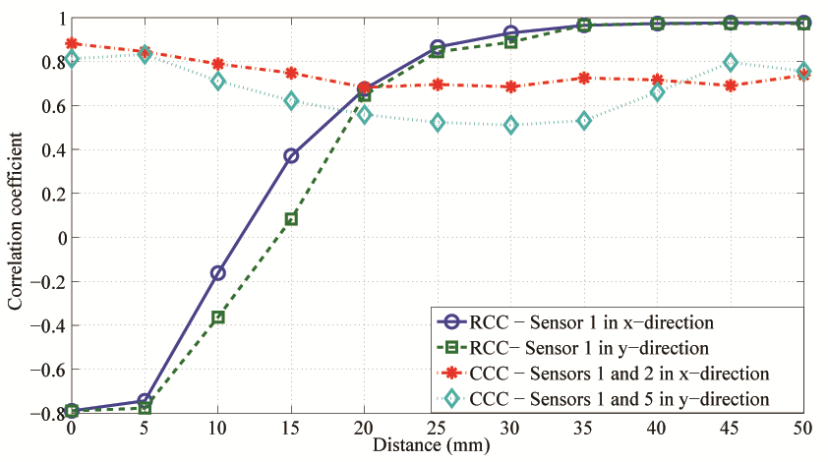

Fig.8 Measured RCC of sensor 1,CCC of sensor 1 and 2 as well as sensor 1 and 5 for one egg being moved in the $x$ direction while keeping $y$ as $0 \mathrm{~mm}$ and fixing $x$ as $0 \mathrm{~mm}$ and moving in $y$ direction

\section{A. Reflection coefficient correlation (RCC)}

Simulated and measured RCC maps for sensor 1, with 1 to 4 egg quantities are depicted in Fig. 9 (a) to (d) respectively in which the horizontal axis denotes the distance the egg moves in the $x$ direction and the vertical the $y$ direction. The correlation falls to a deeper negative value in the measured case $(-0.6$ to -0.8$)$ rather than -0.2 to -0.3 in the simulated case. This is due to the very low magnitude $(-40 \mathrm{~dB})$ at the resonant point of $4 \mathrm{GHz}$ when the egg is present or near the sensor indicated in Fig. 5. The difference in precision when comparing measurement and simulation for such deep resonances results in differently computed low values of correlation. Otherwise, the simulated and measured results are in good agreement where there is higher correlation. The results in Fig. 9 show that low correlations are obtained from the origin of the map regardless of the number of eggs, which is the only position where an egg is covering sensor 1 . At distances beyond $20 \mathrm{~mm}$ from the origin, the correlation tends towards unity beyond the sensor's minimum detectable region found in [18]. Therefore any egg beyond this distance must be determined by another sensor.

Since it was found in Fig. 9 that there is good consistency between measured and simulated RCC maps, only measured RCC maps will be analyzed from this point forward. Measured RCC maps of sensor 2 for three eggs and four eggs are plotted in Fig. 10 (a) and (b) in which two low correlation regions at both the origin $(0,0)$ and at $(50,0)$ can be observed due to the first egg covering sensor 1 and a second egg covering sensor 2 initially. As the eggs translate $50 \mathrm{~mm}$ in the $x$ direction, the first egg from sensor 1 will subsequently cover sensor 2 at $(50,0)$. From this, it is worth noting that with 
up to four eggs, it is possible to distinguish two eggs that cover a single sensor at different points when moving in the $x$ direction. Measured RCC maps of sensor 5 for four eggs are shown in Fig.11. There are now two low correlation regions at $(0,0)$ and $(0,45)$ since a third egg covers sensor 5 initially at $(0,45)$ while the first egg covers sensor 5 as the eggs move in the $y$ direction $45 \mathrm{~mm}$. Therefore it is also possible to discriminate two eggs covering a single sensor moving in the $y$ direction.
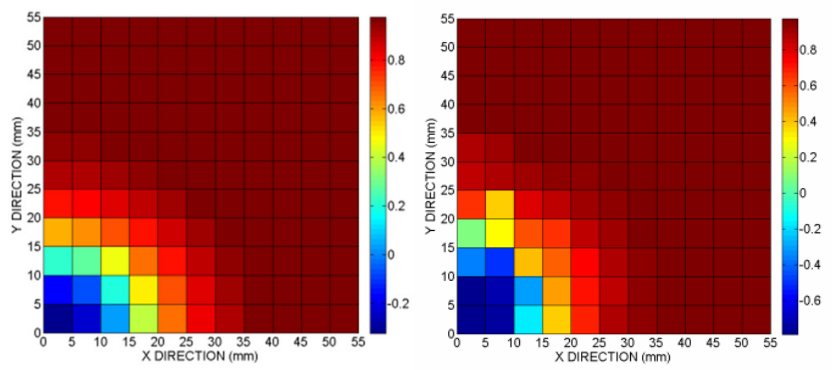

(a)
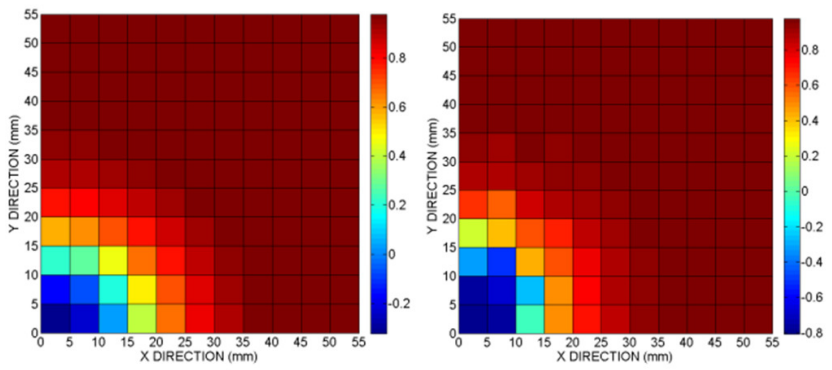

(b)
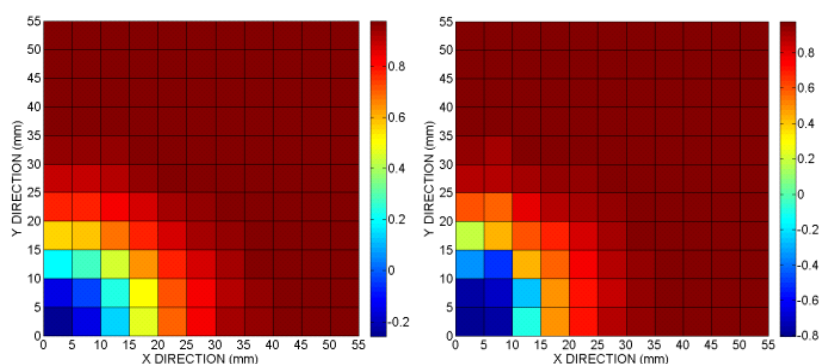

(c)
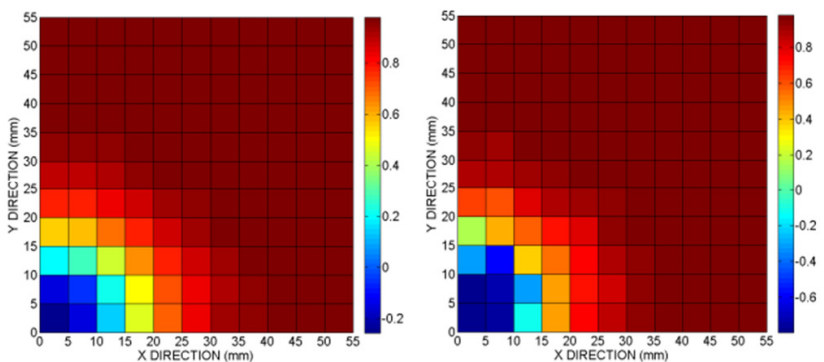

(d)

Fig.9 (Left) Simulated and (Right) measured RCC of sensor 1 at $(0,0)$ with (a) one egg on sensor 1 at $(0,0)$, (b) two eggs starting on sensors 1 and 5 at $(0,0)$ and $(0,45),(\mathrm{c})$ three eggs starting on sensors 1 at $(0,0), 2$ at $(50,0)$ and 5 at $(0,45)$ and $(d)$ four eggs starting over all sensors (i.e. $(0,0),(50,0),(0,45)$ and $(50,45))$

Considering sensor 6, the measured RCC maps for four eggs are shown in Fig. 12. A low correlation coefficient occurs in all four corners indicating the possibility to distinguish each individual egg when it covers a sensor, no matter which direction the eggs are translated.

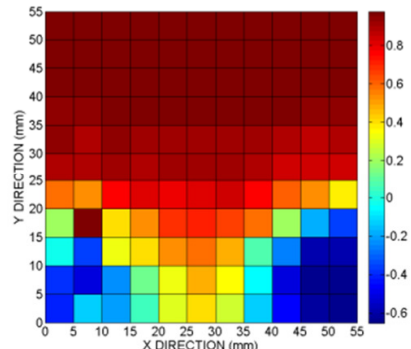

(a)

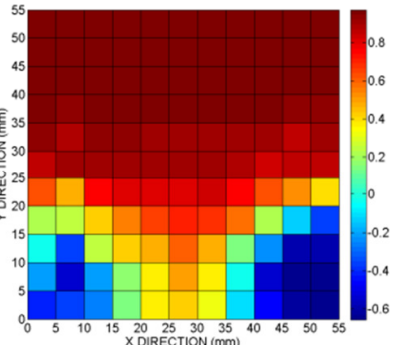

(b)
Fig.10 Measured RCC of sensor 2 at $(50,0)$ with (a) three eggs starting on sensor 1 at $(0,0), 2$ at $(50,0)$ and 5 at $(0,45)$ and (b) four eggs starting over all sensors (i.e. $(0,0),(50,0),(0,45)$ and $(50,45))$

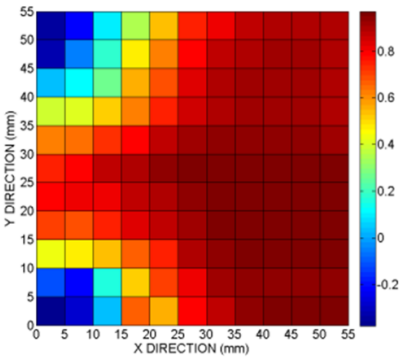

Fig.11 Measured RCC of sensor 5 at $(0,45)$ with four eggs starting over all sensors (i.e. $(0,0),(50,0)$ $(0,45)$ and $(50,45))$

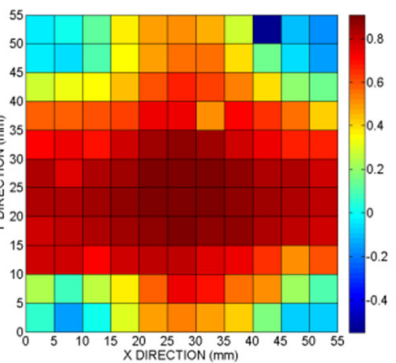

Fig.12 Measured RCC of sensor 6 with four eggs starting over all sensors (i.e. $(0,0),(50,0),(0,45)$ and $(50,45))$
The results presented for RCC are promising in terms of evaluating the presence of an egg where it covers a sensor within $20 \mathrm{~mm}$ radius of its center consistent with the minimum detectable region [18]. This will work well for smart egg boxes where the sensors are fixed below the resting position of each egg. If sensors are not attached to the egg box but on the shelf it is placed, sensors may not always be directly covered by an egg or item, thus RCC will not give enough information about the quantity when items (or eggs) are in between sensors. CCC information then becomes necessary.

\section{B. Coupling coefficient correlation (CCC)}

Simulated and measured CCC maps between sensors 1 and 2 are depicted in Fig. 13 (a) to (d) for 1 to 4 egg quantities respectively. They consider the same configurations as for RCC described in Fig. 7. In Fig. 13 (a), the CCC level drops as low as 0.7 in measurement when the egg is in between the two sensors at $(25,0)$, while the correlation value becomes high when the egg is directly over sensor 1 . However, the overall CCC is no less than 0.85 through simulation. There are three notable reasons contributing to this difference. Firstly, noise in the vector network analyzer (VNA) exists in measurement, where low coupling values are measured, though the effect on correlation is small as measured signal to noise ratio is still sufficient. Secondly, the material for eggs in simulation is considered as homogenous in which the dielectric constant is the same for any point in the egg, however, it is unrealistic for the real egg as it consists of yolk as well as egg white where dielectric values are significantly different [24]. Thirdly, the effect of the sensors' connectors 
are not considered in simulation. It should be also noted that symmetry is not observed for the CCC measurements in the way that it was observed for RCC measurements, which is also owing to precision issues in computation particularly with measurement data.
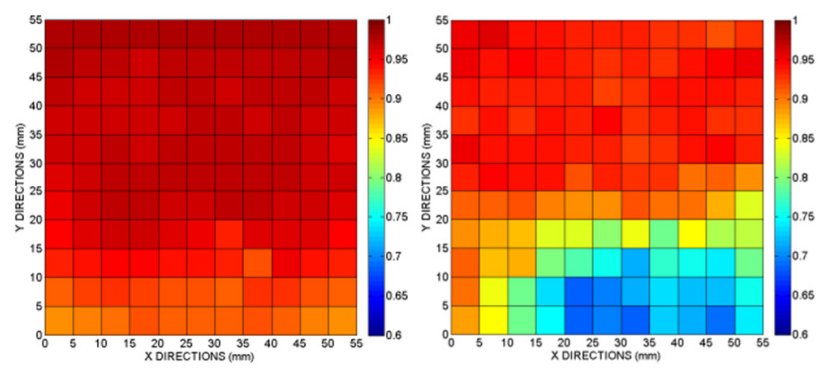

(a)
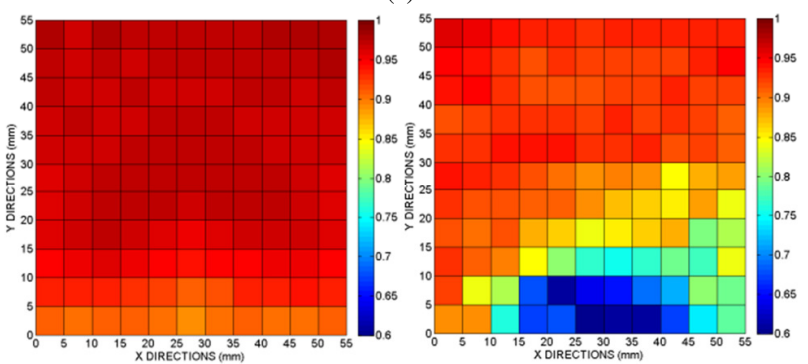

(b)
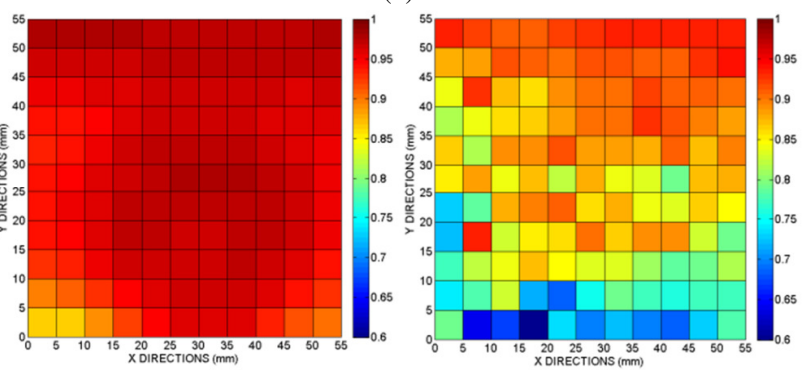

(c)
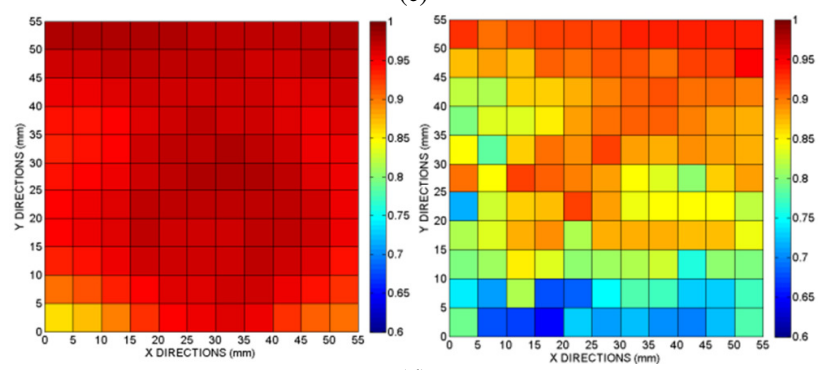

(d)

Fig.13 Simulated (Left) and Measured (Right) CCC of sensor 1 and 2 with (a) one egg starting on sensor 1 at $(0,0)$, (b) two eggs starting on sensor 1 at $(0,0)$ and 5 at $(0,45)$, (c) three eggs starting on sensors 1 at $(0,0), 2$ at $(50,0)$ and 5 at $(0,45)$ and (d) four eggs starting over all sensors (i.e. $(0,0)$, $(50,0),(0,45)$ and $(50,45))$

For the case of one egg in Fig. 13 (a), this result is coupled with the RCC results for the same case in Fig. 9 (a), which enables a single egg to be detected regardless of its position between two adjacent sensors. It is now possible to detect the presence of an egg when placed midway between two sensors. This can be identified clearly in Fig. 8, where at $x=25 \mathrm{~mm}$, the RCC rises above 0.8 but the CCC between sensors 1 and 2 drops to a minimum of 0.7 beyond the stated coherence distance of $20 \mathrm{~mm}$ for $x$ direction. Where $x<20 \mathrm{~mm}$, the RCC of sensor 1 is below 0.7 and thus can be used to detect when the egg is over the sensor, while for $x>30 \mathrm{~mm}$, sensor 2 can be used for the same purpose. Where $20 \mathrm{~mm}<x<30 \mathrm{~mm}$, the CCC is consistently low at 0.7 and therefore a suitably low threshold, for example 0.75 , can detect the presence of an egg in this range using the RCCs of the two sensors and CCC between the two sensors. It can be further noted by analysis of Fig. 9 (a) and 13 (a) that the same criteria can be applied for fixed values of $y$ up to $15 \mathrm{~mm}$ where the edge of the detectable region is reached [18].

The CCC from sensors 1 to 2 is impacted by adding more eggs in Fig. 13 (b) to (d), especially with three and four eggs, a second egg is initially covering sensor 2 at coordinate $(0,0)$. The measured results show that at coordinate $(25,0)$ the CCC still falls below 0.7 , thus still enabling an egg to be detected between two sensors when others are contained in the box.

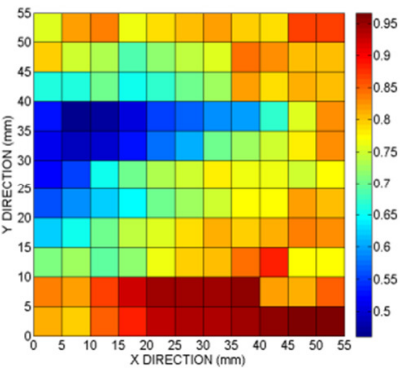

(a)

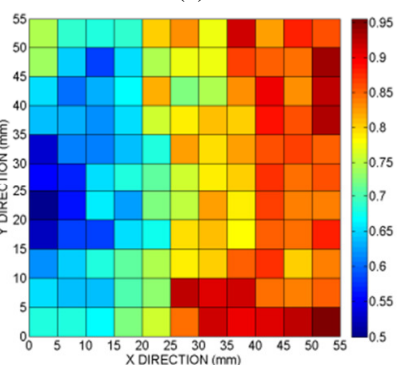

(c)

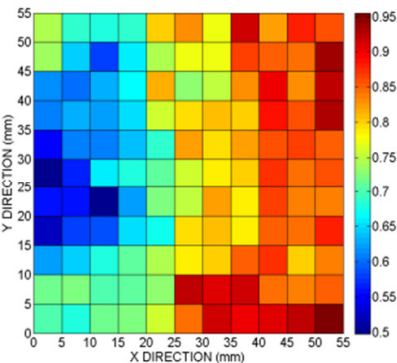

(b)

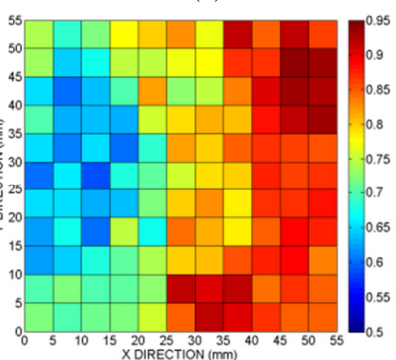

(d)
Fig.14 Measured CCC of sensor 1 and 5 with (a) one egg starting on sensor 1 at $(0,0),($ b) two eggs starting on sensors 1 at $(0,0)$ and 5 at $(0,45),(\mathrm{c})$ three eggs starting on sensors 1 at $(0,0), 2$ at $(50,0)$ and 5 at $(0,45)$ and $(\mathrm{d})$ four eggs starting over all sensors (i.e. $(0,0),(50,0),(0,45)$ and $(50,45))$

As simulation results are not suitable for analyzing CCC, only measured results are presented beyond this point. Fig. 14 considers the CCC between sensors 1 and 5 in the $y$-direction. For a single egg case shown in Fig. 14 (a), the measured CCC drops to a minimum from coordinate $(0,22.5)$. It is at this coordinate that the egg is halfway between the feed points of the two sensors. It can be seen in Fig. 8 that for $y<22.5 \mathrm{~mm}$, the RCC of sensor 1 is below 0.7 , thus can be used to detect the egg when it is close to the sensor in the $y$ direction. Detection of the egg with sensor 5 using RCC is possible when $y>35 \mathrm{~mm}$ as shown in Fig. 11. For the region when $22.5 \mathrm{~mm}<y<35 \mathrm{~mm}$, RCC rises above 0.8 , however, the $\mathrm{CCC}$ as shown in Fig. 8 is lower than 0.6 in this region, which can be used to sense the presence of egg again using a suitable threshold such as 0.7 (also beyond the earlier stated coherence distance of $10 \mathrm{~mm}$ in the $y$ direction). The presence of a second egg on sensor 5 in Figs. 13 (b), (c) and (d) due to using more eggs does not provide any significant effect in measurement where $\mathrm{CCC}$ in the region of interest has a 
consistently low value from 0.5 to 0.6 . The drop in correlation midway between sensors 1 to 5 is also consistent for fixed values of $x$ up to $20 \mathrm{~mm}$ where the edge of the detectable region is reached [18].

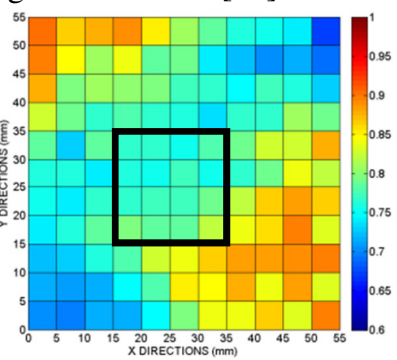

(a)

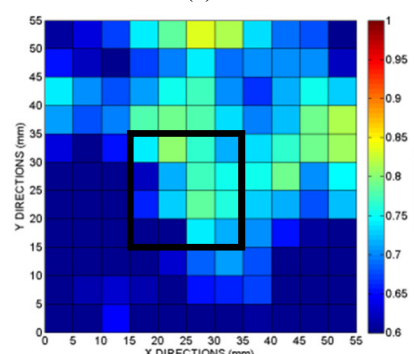

(c)

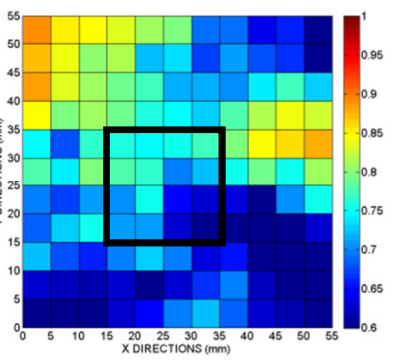

(b)

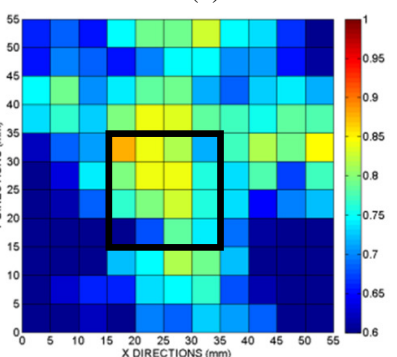

(d)
Fig. 15 Measured CCC of sensor 1 and 6 with (a) one egg starting on sensor 1 at $(0,0),($ b) two eggs starting on sensors 1 at $(0,0)$ and 5 at $(0,45),(\mathrm{c})$ three eggs starting on sensors 1 at $(0,0), 2$ at $(50,0)$ and 5 at $(0,45)$ and $(d)$ four

eggs starting over all sensors (i.e. $(0,0),(50,0),(0,45)$ and $(50,45))$

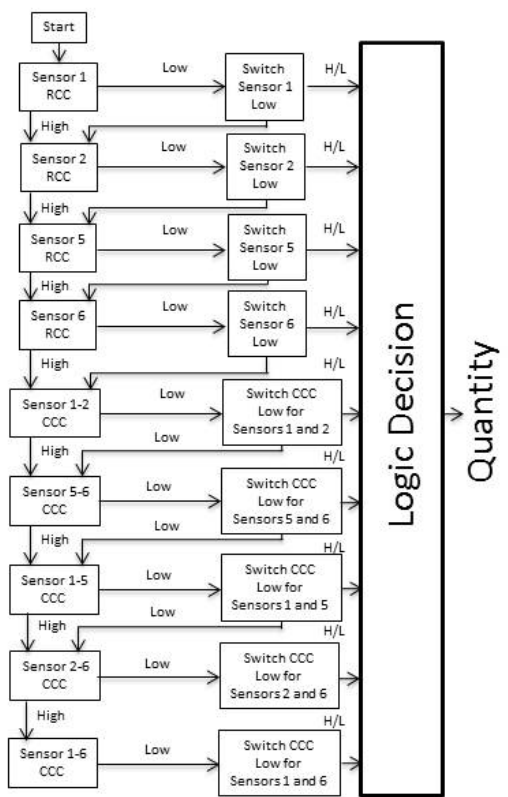

Fig. 16 Flow Diagram Illustration of the logic decision process for detecting eggs within four sensors, 1, 2, 5 and 6

Fig. 15 shows results of the CCC between sensors 1 and 6, which are diagonally opposite each other. In the case of a single egg in Fig. 15 (a), the measured correlation map is somewhat different to the case of linearly separated elements due to highly different coupling characteristics that change more when an egg is placed above sensors 1 or 6 , yielding the lowest correlation at $(0,0)$ and $(50,45)$ below 0.9 . The level of correlation below this threshold is, however, still maintained in the diagonal path between sensors 1 and 6 , in particular in the region outlined by the bold lined square. In this region, the CCC information must be used for detection where neither the RCC of a single sensor or the CCC of horizontally or vertically spaced sensors can be used. In such cases, the RCC of either diagonally separated sensor is above 0.85 and therefore an egg can be detected within this region where RCC meets this condition and the CCC falls below 0.9. Figs. 15 (b)-(d) show that this requirement is maintained with the addition of further eggs. Lower correlation maps emerge as more eggs are added, particularly with three and four eggs, which is expected as they substantially reduce coupling between sensors 1 and 6 . The diagonal spacing of sensors 1 and 6 have been chosen in this case for analysis though the same principle can equally apply to sensors 2 and 5, which are diagonally separated. Clearly in this instance the correlation maps will be mirrored with the line of symmetry from $(27.5,0)$ to $(27.5,55)$.

As a conclusion of all the results presented in this section, by encompassing both RCC and CCC parameters, there is a possibility to detect the quantity of up to four eggs regardless of the position of a single egg within the region of four sensors making a $2 \times 2$ array. The process of how the egg quantity is computed is shown in the flow diagram in Fig. 16. First the four RCC and the five CCC values are calculated and then a logic decision is made with the nine states being high (H) or low (L). Examples of logic decision tables with appropriate notes are given in the appendix, which could be also considered as truth tables. The logic decision could be re-mapped to overlapping adjacent sets of four sensors to detect more eggs on larger arrays. These results prove the egg quantities can be detected either with sensors attached to the package or placing the package over an array of dedicated sensors assuming no other obstructing clutter is present. The effect of clutter is discussed in the next section.

\section{REACTIVE NEAR FIELD EGG DETECTION IN CLUTTERED CONDITIONS}

The study of the effect of other food or clutter on top of or around the egg box is analyzed in this section. Such evaluation requires an empirical set of measurements, where ten types of food including fruits, vegetables, bread and liquids are randomly combined on top of or around the egg box after which the egg detection is compared both with and without the clutter present. Only a single egg is used to evaluate the impact of other clutter here as the RCC and CCC have proved that if it is feasible for a single egg case, then it is also validated when additional eggs are present. Three metrics are compared in this study, which are reflection coefficient correlation (RCC) and the coupling correlation coefficient (CCC) between two sensors in both $x$ and $y$ directions.

A schematic plot of the measurement setup up is shown in Fig. 17 in which the egg box was surrounded by other food while containing one egg above sensor 10 in the first instance. Another instance is to consider the effects when the egg is halfway between sensors 10 and 14. S-parameters of sensor $10, s_{1010}$, sensor $14, s_{1414}$ and the coupling coefficient between two adjacent sensors, $s_{1011}$ and $s_{1014}$ are measured in each test 
with and without the food clutter.

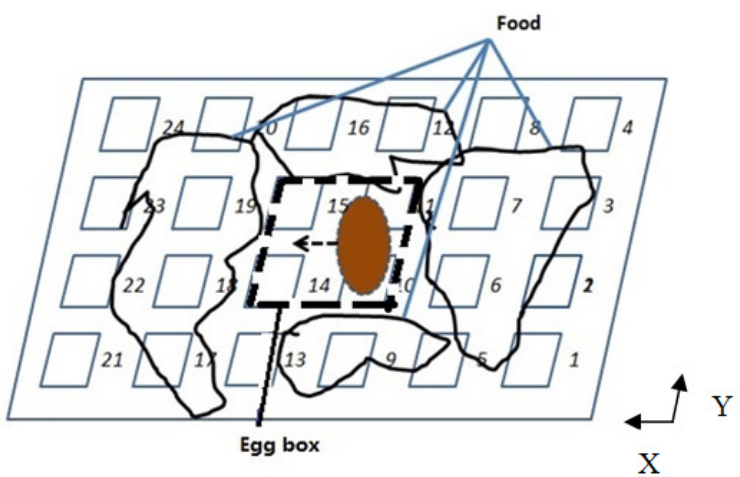

Fig.17 Schematic plot of egg detection in the general clutter scenarios (Dashed line: the position of egg box)

In order to directly compare the effect of clutter and separate it from the effect of the eggs themselves, a correlation coefficient is defined to compare measured S-parameters in the cluttered and clutter free cases as follows:

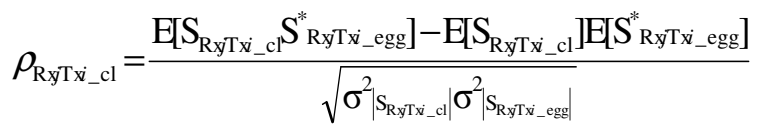

where $\mathrm{S}_{\mathrm{Rx} j \mathrm{Txi} i \mathrm{cl}}$ denotes the $\mathrm{S}$-parameter between transmitter $i$ and receiver $j$ (i.e. $\mathrm{s}_{j i}$ ) in the presence of the egg with clutter while $\mathrm{S}_{\mathrm{Rxj} \text { Txi_egg }}$ denotes parameter $\mathrm{s}_{j i}$ with the egg only. Furthermore, in order to better interpret the variation involved in different scenarios quantitatively, the coefficient of variation $(\mathrm{CV})$ is introduced which is a measure of the variability of a series of numbers [26]. It has the unique advantage to compare two sets of data with different measures, which is suitable for observation of the variation of RCC and CCC caused by the ten different scenarios. The expression of CV can be written as [26]:

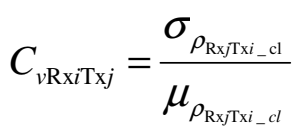

where $C_{v \mathrm{Rx} i \mathrm{Tx} j}$ is a coefficient of variation related to the correlation coefficient between $j^{\text {th }}$ transmitter and $i^{\text {th }}$ receiver and usually expressed in percentage format, while $\sigma_{\rho \mathrm{Rx} j \mathrm{Tx} i \mathrm{cl}}$ and $\mu_{\rho \mathrm{Rxj} \text { Txicl }}$ are the standard deviation and mean of the recorded correlation coefficient values of ten different scenarios.

\section{A. Effect of food clutter around the egg box}

Ten chosen food combinations to reflect real fridge usage scenarios are given in Table I and photographs of some of the scenarios where food was placed around the egg box are shown in Fig. 18 (a)-(d). The clutter effects on RCC $\left(\rho_{\mathrm{Rx} 10 \mathrm{Tx} 10 \_\mathrm{cl}}, \rho_{\mathrm{Rx} 11 \mathrm{Tx} 11 \_\mathrm{cl}}\right.$ and $\left.\rho_{\mathrm{Rx} 14 \mathrm{Tx} 14 \text { cl }}\right)$ and $\mathrm{CCC}\left(\rho_{\mathrm{Rx} 11 \mathrm{Tx} 10 \_\mathrm{cl}}\right.$ and $\left.\rho_{\mathrm{Rx} 14 \mathrm{~T} \times 10 \_c 1}\right)$ were evaluated using equation (8) with an egg above sensor 10 . High correlation, above 0.9 was observed in RCC cases, $\rho_{\mathrm{Rx} 11 \mathrm{Tx11} \text { cl, }} \rho_{\mathrm{Rx} 14 \mathrm{~T} \times 14 \_c l}$ and also for CCC case,

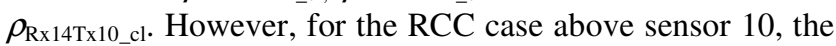

correlation $\rho_{\mathrm{Rx} 10 \mathrm{Tx} 10 \mathrm{cl}}$ fell below 0.9 . This is reflected in Table II where the CV for this case has the highest value above sensor 10 , which is $3 \%$. This indicates that though the clutter has the most effect on the RCC of sensor 10 when the egg is above the sensor, such effect is negligible because the $\mathrm{RCC}$ itself is already at its lowest value. Finally, $\rho_{\mathrm{Rx} 11 \mathrm{Tx} 10_{\mathrm{c}} \mathrm{cl}}$ falls frequently below 0.9 though the $\mathrm{CCC}$ in this instance is not of interest as the egg is covering a single sensor and only $\mathrm{RCC}$ is required for detection. Therefore egg detection when over a sensor is not affected largely because the egg box "protects" the sensor.

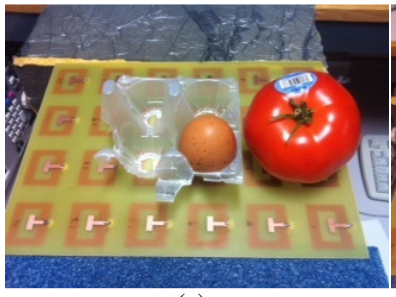

(a)

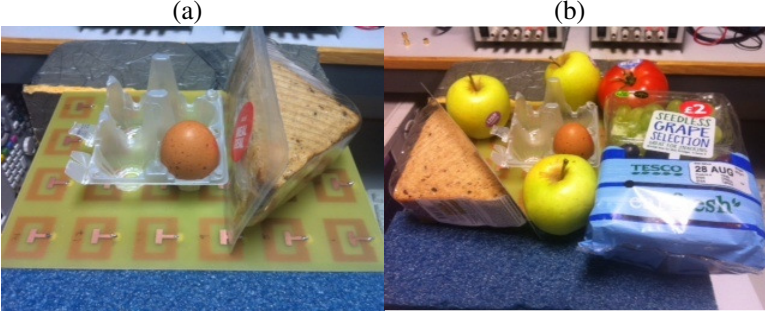

(c)

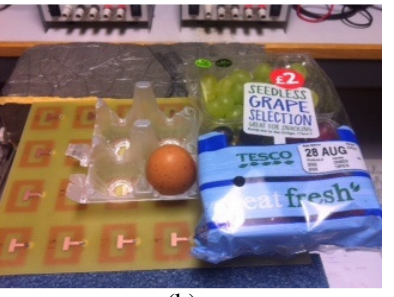

(d)
Fig.18. Photograph of (a) scenario 1 (b) scenario 2 (c) scenario 3 (d) scenario 9 when food placed around the egg box TABLE I

DIFFERENT FOOD SCENARIOS FOR CLUTTER STUDY

\begin{tabular}{cccc}
\hline \hline $\begin{array}{c}\text { Scenario } \\
\text { Number }\end{array}$ & Food & $\begin{array}{c}\text { Scenario } \\
\text { Number }\end{array}$ & Food \\
\hline 1 & Tomato & 6 & Butter, Cheese \\
2 & Grapes & 7 & Yogurt, Cheese \\
3 & Sandwich & 8 & Water \\
4 & Apple & 9 & $1,2,3$ and 4 \\
5 & Bread & 10 & $1,3,7$ and 8 \\
\hline \hline
\end{tabular}

\begin{tabular}{|c|c|c|c|c|c|}
\hline TOPOLOGY & $\begin{array}{c}C_{v} \\
\operatorname{Rx} 10 \\
\operatorname{Tx} 10 \\
\end{array}$ & $\begin{array}{c}C_{v} \\
\operatorname{Rx} 11 \\
\operatorname{Tx} 10 \\
\end{array}$ & $\begin{array}{c}C_{v} \\
\operatorname{Rx} 14 \\
\text { Tx } 10 \\
\end{array}$ & $\begin{array}{c}C_{v} \\
\operatorname{Rx} 11 \\
\operatorname{Tx} 11 \\
\end{array}$ & $\begin{array}{c}C_{v} \\
\operatorname{Rx} 14 \\
\operatorname{Tx} 14 \\
\end{array}$ \\
\hline $\begin{array}{c}\text { Above } \\
\text { sensor } 10\end{array}$ & $3 \%$ & $1.88 \%$ & $1.86 \%$ & $0.74 \%$ & $0.09 \%$ \\
\hline $\begin{array}{c}\text { Between } \\
\text { sensor } 10 \\
\text { and } 14\end{array}$ & $1.09 \%$ & $9.86 \%$ & $2.12 \%$ & $1.43 \%$ & $0.95 \%$ \\
\hline
\end{tabular}

TABLE II

The same correlation values for RCC and CCC cases were evaluated for the ten clutter scenarios when an egg is in between sensors 10 and 14 and the corresponding CV values are also shown in Table II. In such a position, the parameters $\rho_{\mathrm{Rx} 10 \mathrm{Tx} 10 \_\mathrm{cl}}, \rho_{\mathrm{Rx} 14 \mathrm{Tx} 10 \_\mathrm{cl}}$ and $\rho_{\mathrm{Rx14Tx14} \text { cl }}$ are of great interest where the correlation was above 0.9 in all cases and $\mathrm{CV}$ is less than $2.2 \%$, thus will not affect detection of the egg in this position. Furthermore, $\rho_{\mathrm{Rx} 11 \mathrm{Tx} 11_{\text {cl }} \text {, }}$ though not relevant in this case, was also always higher than 0.9 with a $\mathrm{CV}$ of $1.43 \%$. However, $\rho_{\mathrm{Rx} 11 \mathrm{~T} \times 10 \mathrm{cl}}$ dropped as low as 0.59 , with a corresponding $\mathrm{CV}$ of $9.86 \%$. The latter case is expected as the 
clutter will directly affect coupling between sensors 10 and 11 , where the detectable region [18] is exposed and not covered by the box, which could in some instances cause false egg detection. The worst instance of this is in scenario 8 where water is placed next to the egg box. This can be overcome if necessary by deployment of smaller size sensors with higher density.

\section{B. Effect of food clutter above the egg box}

It is also assumed that food can be above the egg box in a cluttered fridge, thus the ten scenarios described in Table I were tested with the corresponding food items placed above the egg box, some of which are illustrated in Fig. 19 (a)-(d).
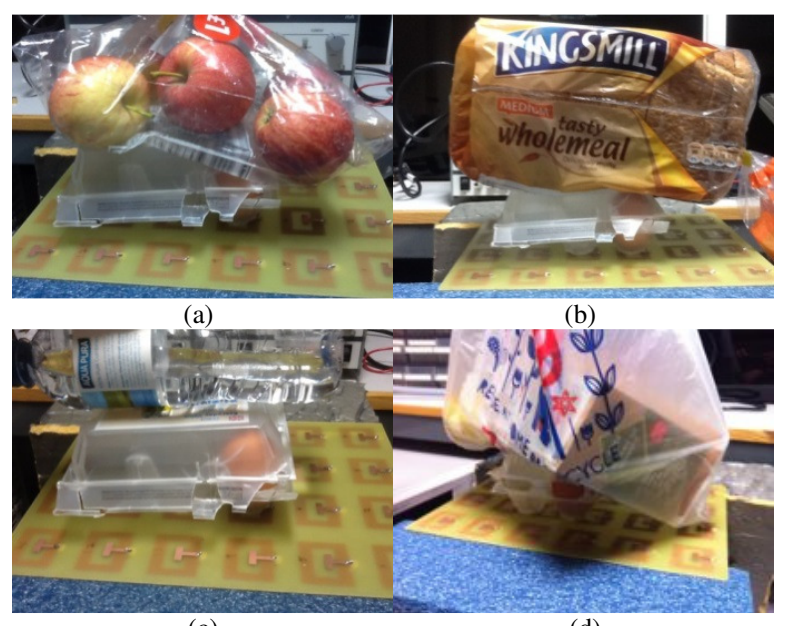

(c)

(d)

Fig. 19 Photograph of (a) scenario 4 (b) scenario 5 (c) scenario 8 (d) scenario 10 when food placed above the egg box

TABLE III

SUMMARY OF CV WITH FOOD CLUTTER ABOVE THE BOX FOR $\rho_{\mathrm{RXITXJCL}}$

\begin{tabular}{cccccc}
\hline \hline TopoloGy & $\begin{array}{c}C_{v} \\
\mathrm{Rx} \mathrm{10} \\
\mathrm{Tx} 10\end{array}$ & $\begin{array}{c}C_{v} \\
\mathrm{Rx} 11 \\
\mathrm{Tx} 10\end{array}$ & $\begin{array}{c}C_{v} \\
\mathrm{Rx} \mathrm{14} \\
\mathrm{Tx} 10\end{array}$ & $\begin{array}{c}C_{v} \\
\mathrm{Rx} 11 \\
\mathrm{Tx} 11\end{array}$ & $\begin{array}{c}C_{v} \\
\mathrm{Rx} 14 \\
\mathrm{Tx} 14\end{array}$ \\
\hline $\begin{array}{c}\text { Above } \\
\text { sensor 10 }\end{array}$ & $1.18 \%$ & $3.27 \%$ & $1.24 \%$ & $0.29 \%$ & $0.32 \%$ \\
$\begin{array}{c}\text { Between } \\
\text { sensor 10 } \\
\text { and 14 }\end{array}$ & $0.94 \%$ & $7.11 \%$ & $4.06 \%$ & $0.83 \%$ & $1.37 \%$ \\
\hline \hline
\end{tabular}

The same five correlation values for RCC and CCC cases around the egg box were evaluated for food above the egg box and the corresponding $\mathrm{CV}$ values are shown in Table III. When the egg is directly above sensor 10 , the presence of other food above the eggs has negligible effect on all five parameters, which had values above 0.9 , except $\rho_{\mathrm{Rx11Tx10} \text { cl }}$, which fell as low as 0.83 and had a high $\mathrm{CV}$ above $3 \%$. As before, however, this parameter is not relevant when the egg is covering sensor 10 as only RCC values are of interest. When the egg is midway between sensors 10 and 14, the parameter $\rho_{\mathrm{Rx} 14 \mathrm{Tx} 10 \text { cl }}$ dropped as low as 0.83 , though this effect is negligible since the CCC will already be low and the egg will be successfully detected while the RCC values of sensors 10 and 14 are suitably high. Similar to the case with clutter around the egg box, $\rho_{\mathrm{Rx} 11 \mathrm{Tx} 10 \mathrm{cl}}$ was found to vary more significantly than any other parameter and fell as low as 0.78 in scenario 9, with a $\mathrm{CV}$ of $3.27 \%$, where the clutter is so great it is actually also surrounding the egg box. The other nine scenarios had less impact than this with $\rho_{\mathrm{Rx} 11 \mathrm{Tx} 10 \_\mathrm{cl}}$ above 0.9 meaning clutter strictly above the egg box is not vulnerable to causing detection error.

The results presented in this section therefore prove the case that clutter will not cause detection failure in a smart package where the sensors are either fixed to the package or that the package is correctly placed over dedicated sensors on a shelf. It must also be assumed that only the intended items (eggs in this case study) are placed in the package.

\section{CONCLUSION AND ANALYSIS OF CONCEPT}

The study of UWB reactive near field detection as an enabling technology for smart packaging with a wide range of applications has been carried out using an example case of eggs in a box. The presence of eggs and their quantity can be determined by analyzing the filtering of a UWB impulse using monostatic and bistatic detection modes depending on where the egg is positioned in relation to the sensors. Correlation coefficients have been derived as metrics for detection, which show that without the presence of clutter, eggs can be detected either directly above a sensor or in between two sensors. Hence the concept is proven for both sensors attached to a smart package, or where the package is placed randomly on an array of sensors on a shelf. Resilience to clutter is maintained under the assumption that sensors are always positioned directly under the slots of the package in which items are placed and that it is known what items are placed in the package.

The concept could be scaled to larger dielectric items by scaling the sensors up in size and down in frequency in order to change the detectable region to half that of the distance between sensors, which was $25 \mathrm{~mm}$ for an egg box. However, if the sensors are fixed directly below their respective slots in a package then scaling is not necessary as only low RCC is required for detection. For smaller items, scaling sensors down in size and up in frequency is necessary and thus the concept is limited by how small a size is practical.

\section{APPENDIX - EGG POSITION SCENARIOS AND DETECTION LOGIC}

This appendix lists selected detection scenarios that could be required for four individual sensors arranged in a square to demonstrate how logic combinations resolved in Fig. 16 would be represented. All possible combinations are not included due to limited space. Table A1 demonstrates a single egg case on sensor 1 , between sensors 1 and 5 , between sensors 1 and 2 and in the center. $\mathrm{H}$ denotes a high correlation and $\mathrm{L}$ a low correlation below a set threshold.

Table A2, gives four examples where two eggs are present. The first two cases are simple whereby the two eggs are placed over two sensors directly or between two sensors. The remaining two scenarios consider how one egg is placed over a sensor and the other is in between two sensors for which unique logic states exist.

It is noteworthy that two eggs could be positioned where one egg is between two sensors and the other is on the outer 
edge. Three examples are given in Fig. A1. Where an egg is on the edge, another sensor is required, but the same principles can re-mapped onto a new set of four sensors that overlap with the sensors in these examples.

TABLE A1

LOGIC DECISIONS FOR DETECTING A SINGLE EGG

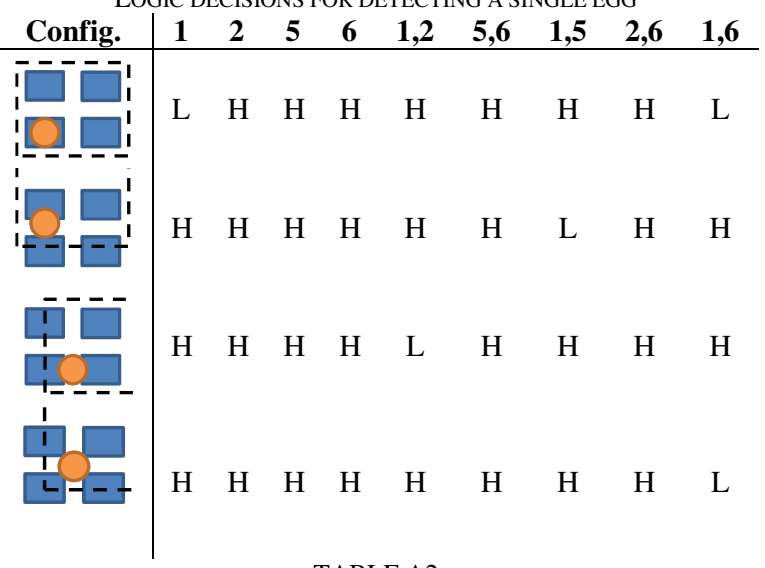

TABLE A2

LOGIC DECISIONS FOR DETECTING TWO EGGS

\begin{tabular}{|c|c|c|c|c|c|c|c|c|c|}
\hline \multirow[t]{5}{*}{ Config } & 1 & 2 & 5 & 6 & 1,2 & 5,6 & 1,5 & 2,6 & 1,6 \\
\hline & $\mathrm{L}$ & $\mathrm{H}$ & $\mathrm{L}$ & $\mathrm{H}$ & $\mathrm{H}$ & $\mathrm{H}$ & $\mathrm{H}$ & $\mathrm{H}$ & $\mathrm{L}$ \\
\hline & $\mathrm{H}$ & $\mathrm{H}$ & $\mathrm{H}$ & $\mathrm{H}$ & $\mathrm{L}$ & $\mathrm{L}$ & $\mathrm{H}$ & $\mathrm{H}$ & $\mathrm{L}$ \\
\hline & $\mathrm{L}$ & $\mathrm{H}$ & $\mathrm{H}$ & $\mathrm{H}$ & $\mathrm{H}$ & $\mathrm{L}$ & $\mathrm{H}$ & $\mathrm{H}$ & $\mathrm{L}$ \\
\hline & $\mathrm{L}$ & $\mathrm{H}$ & $\mathrm{H}$ & $\mathrm{H}$ & $\mathrm{H}$ & $\mathrm{H}$ & $\mathrm{H}$ & $\mathrm{L}$ & $\mathrm{L}$ \\
\hline
\end{tabular}

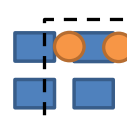

(a)

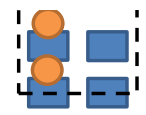

(b)

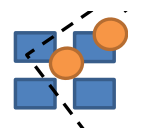

(c)
Fig. A1 Three example scenarios of two eggs with one positioned on the sensor edges.

\section{REFERENCES}

[1] M. Darianian and M.P. Michael, "Smart home mobile RFID-based Internet-of-Things systems and services," in International Conference on Advanced Computer Theory and Engineering, Phuket, 2008, pp. 116-120.

[2] L. Xie, Y. Yin, X. Lu, B. Sheng, and S. Lu, "iFridge: an intelligent fridge for food management based on RFID technology," in UbiComp, Zurich, 2013, pp. 291-294.

[3] S. Luo, J.S. Jin, and J. Li, "A smart fridge with an ability to enhance health and enable better nutrition," International Journal of Multimedia and Ubiquitous Engineering, vol. 4, no. 2, pp. 69-80, Apr 2009.

[4] H. Gu and D. Wang, "A content-aware fridge based on RFID in smart home for home-healthcare," in 11th Internation Conference on Advanced communication technology, Phoenix Park, 2009, pp. 987-990.

[5] Y.C. Chen, M.F. Hiseh, C.C. Wang, and H.R. Lee, "RFID-based intelligent systems for home-healthcare," in International Conference on Consumer Electronics, Las Vegas, 2007, pp. 1-2.
[6] Z. Ding, J. Li, and B. Feng, "Radio frequency identification in food supervision," in International conference on Advanced Communcation Technology, Gangwon-Do, 2007, pp. 542-545.

[7] E.C. Fear, X. Li, S.C. Hagness, and M.A. Stuchly, "Confocal microwave imaging for breast cancer detection: localization of tumours in three dimensions," IEEE Transactions on Biomedical Engineering, vol. 49, no. 8, pp. 812-822, Aug 2002.

[8] Y. Yang and A.E. Fathy, "See-through-wall imaging using ultra wideband short-pulse radar system," in IEEE Antennas and Propagation Society International Symposium, Washington, 2005, pp. 334-337.

[9] L. Carin, N. Geng, M. McClure, J. Sichina and L. Nguyen, "Ultra wideband synthetic-aperture radar for mine filed detection," IEEE on Antennas and Propagation Magazine, vol. 41, no. 1, pp. 18-33, Feb 1999.

[10] C.E. Baun, "On the singularity expansion method for the solution of electromagnetic interaction problem," Air Force Weapons Lab, Interaction Notes 1971.

[11] W.M. Boemer, W. Yan, A.Q. Xi, and Y. Yamaguchi, "On the basic principles of radar polarimetry: the target characteristic polarization state theory of Kennaugh, Huynen's polarization fork concept and its extension to the partially polarized case," Proceedings of the IEEE, vol. 79 , no. 10 , pp. 1538-1550, Oct 1991.

[12] C.E. Baun, E.J. Rothwell, Y.F. Chen, and D.P. Nyquist, "The singularity expansion method and its application to target identification," Proceedings of the IEEE, vol. 79, no. 10, pp. 1481-1492, Oct 1991.

[13] A. Mroue, M. Heddebaut, F. Elgahhar, A. Rivenq, and J.M. Rouvaen, "UWB radar and leaky waveguide for fall on track object identification," , Washington, 2010, pp. 573-577.

[14] H. Ma and T.W.C. Brown, "Egg quantity determination based on characteristic polarisation states," IET Electronics Letters, vol. 48, no. 9, pp. 472-474, Apr 2012

[15] F.Aldhubaib, N.V.Shuley and H.S.Lui, "Characteristic polarisation states in an ultrawideband context based on singularity expansion method," IEEE Transactions on Geoscience and Remote Sensing Letters, vol.6, no.4, pp.792-796, Aug 2009.

[16] F.Aldhubaib and N.V.Shuley, "Characteristic polarisation states estimation in an ultra wideband context: A frequency approach," IEEE Transactions on Geoscience and Remote Sensing, vol.47, no.8, pp.2808-2817, Apr 2009.

[17] F.Aldhubaib and N.V.Shuley, "Radar target recognition based on modified characteristic polarisation states," IEEE Transactions on Aersospace and Electronic Systems, vol.46, no.4, Dec 2010.

[18] H. Ma, T.W.C Brown, and A.T.S Ho, "UWB Near Field Detection of Egg Quantity in a Fridge: A Preliminary Study," in Loughborough Antennas and Propagation Conference (LAPC), Loughborough, 2013, pp. 246-249.

[19] Y. Lin and K. Huang, "Compact ultra wideband rectangular aperture antenna and band-notched designs," IEEE Transactions on Antennas and Propagation, vol. 54, no. 11, pp. 3075-3081, Nov 2006.

[20] P. K. Patra, P. D. Calvert, S. B. Warner, "Textile Based Carbon Nanostructured Flexible Antenna," NTC Project No: M06-MD01, 2006.

[21] A.G. Herve, J. Tang, L. Luedecke, and H. Feng, "Dielectric properties of cottage cheese and surface treatment using microwaves," Journal of Food Engineering, vol. 37, no. 4, pp. 389-410, Sep 1998.

[22] J. Wang, J. Tang, Y. Wang, and S. Barry, "Dielectric properties of egg white and whole eggs as influenced by thermal treatments," Elsevier on LWT-Food Science and Technology, vol. 42, no. 7, pp. 1204-1212, Sep 2009.

[23] W. Guo, S. Trabelsi, SO Nelson, and DR Hones, "Storage effects on dielectric properties of eggs from 10 to $1800 \mathrm{MHz}$," Journal of Food Science, vol. 72, no. 5, pp. 335-340, Jun 2007.

[24] A.S. Willsky, A.V. Oppenheim, and S.H. Nawad, Signals and Systems, 2nd ed. New York, United States: Prentice Hall, 1997.

[25] S.Kannan, S.R.S.Dev, Y.Gariepy and G.S.V.Raghavan, "Effect of Radiofrequency heating on the dielectric and physical properties of eggs," Progress in Electromagnetic Research B, vol.51, pp.201-220, 2013.

[26] H. Abdi, "Coefficient of variation," in Encylopedia of research design. United States: SAGE Publications, 2010, pp. 170-172. 